\title{
The hybrid steepest descent method for solving variational inequality over triple hierarchical problems
}

\author{
Nopparat Wairojjana', Thanyarat Jitpeera² and Poom Kumam ${ }^{1 *}$
}

"Correspondence:
poom.kum@kmutt.ac.th
'Department of Mathematics,
Faculty of Science, King Mongkut's
University of Technology Thonburi
(KMUTT), Bangmod, Thung Khru,
Bangkok, 10140, Thailand
Full list of author information is
available at the end of the article

available at the end of the article

\begin{abstract}
An explicit algorithm is introduced to solve the monotone variational inequality over a triple hierarchical problem. The strong convergence for the proposed algorithm to the solution is guaranteed under some assumptions. Our results extend those of liduka (Nonlinear Anal. 71:e1292-e1297, 2009), Marino and Xu (J. Optim. Theory Appl. 149(1):61-78, 2011), Yao et al. (Fixed Point Theory Appl. 2011:794203, 2011) and other authors.

MSC: 46C05; 47H06; 47H09; 47H10; 47J20; 47J25; 65J15

Keywords: nonexpansive mapping; strongly monotone mapping; strongly positive linear bounded operator; Lipchitz continuous; variational inequality; hierarchical fixed point
\end{abstract}

\section{Introduction}

Let $C$ be a closed convex subset of a real Hilbert space $H$ with the inner product $\langle\cdot, \cdot\rangle$ and the norm $\|\cdot\|$. We denote weak convergence and strong convergence by notations $\rightarrow$ and $\rightarrow$, respectively. A mapping $A$ is a nonlinear mapping. The Hartmann-Stampacchia variational inequality [1] for finding $x \in C$ such that

$$
\langle A x, y-x\rangle \geq 0, \quad \forall y \in C .
$$

The set of solutions of (1.1) is denoted by $\operatorname{VI}(C, A)$. The variational inequality has been extensively studied in the literature $[2,3]$.

Let $f: C \rightarrow C$ be called a $\rho$-contraction if there exists a constant $\rho \in[0,1)$ such that

$$
\|f(x)-f(y)\| \leq \rho\|x-y\|, \quad \forall x, y \in C .
$$

A mapping $T: C \rightarrow C$ is said to be nonexpansive if

$$
\|T x-T y\| \leq\|x-y\|, \quad \forall x, y \in C .
$$

A point $x \in C$ is a fixed point of $T$ provided $T x=x$. Denote by $F(T)$ the set of fixed points of $T$; that is, $F(T)=\{x \in C: T x=x\}$. If $C$ is bounded closed convex and $T$ is a nonexpansive mapping of $C$ into itself, then $F(T)$ is nonempty [4].

(c) 2012 Wairojiana et al.; licensee Springer. This is an Open Access article distributed under the terms of the Creative Commons Attribution License (http://creativecommons.org/licenses/by/2.0), which permits unrestricted use, distribution, and reproduction in any medium, provided the original work is properly cited. 
We discuss the following variational inequality problem over the fixed point set of a nonexpansive mapping (see [5-13]), which is called a hierarchical problem. Consider a monotone, continuous mapping $A: H \rightarrow H$ and a nonexpansive mapping $T: H \rightarrow H$. Find $x^{*} \in V I(F(T), A)=\left\{x^{*} \in F(T):\left\langle A x^{*}, x-x^{*}\right\rangle \geq 0, \forall x \in F(T)\right\}$, where $F(T) \neq \emptyset$. This solution set is denoted by $\Xi$.

We introduce the following variational inequality problem over the solution set of the variational inequality problem over the fixed point set of a nonexpansive mapping (see [14-18]), which is called a triple hierarchical problem. Consider an inverse-strongly monotone $A: H \rightarrow H$, a strongly monotone and Lipschitz continuous $B: H \rightarrow H$ and a nonexpansive mapping $T: H \rightarrow H$. Find $x^{*} \in V I(\Xi, B)=\left\{x^{*} \in \Xi:\left\langle B x^{*}, x-x^{*}\right\rangle \geq 0, \forall x \in \Xi\right\}$, where $\Xi:=V I(F(T), A) \neq \emptyset$.

A mapping $A: H \rightarrow H$ is said to be monotone if

$$
\langle A x-A y, x-y\rangle \geq 0, \quad \forall x, y \in H .
$$

A mapping $A: H \rightarrow H$ is said to be $\alpha$-strongly monotone if there exists a positive real number $\alpha$ such that

$$
\langle A x-A y, x-y\rangle \geq \alpha\|x-y\|^{2}, \quad \forall x, y \in H .
$$

A mapping $A: H \rightarrow H$ is said to be $\beta$-inverse-strongly monotone if there exists a positive real number $\beta$ such that

$$
\langle A x-A y, x-y\rangle \geq \beta\|A x-A y\|^{2}, \quad \forall x, y \in H .
$$

A mapping $A: H \rightarrow H$ is said to be L-Lipschitz continuous if there exists a positive real number $L$ such that

$$
\|A x-A y\| \leq L\|x-y\|, \quad \forall x, y \in H .
$$

A linear bounded operator $A$ is said to be strongly positive on $H$ if there exists a constant $\bar{\gamma}>0$ with the property

$$
\langle A x, x\rangle \geq \bar{\gamma}\|x\|^{2}, \quad \forall x \in H
$$

In 2009, Iiduka [14] introduced an iterative algorithm for the following triple hierarchical constrained optimization problem. The sequence $\left\{x_{n}\right\}$ defined by the iterative method below, with the initial guess $x_{1} \in H$, is chosen arbitrarily,

$$
\left\{\begin{array}{l}
y_{n}=T\left(x_{n}-\lambda_{n} A_{1} x_{n}\right), \\
x_{n+1}=y_{n}-\mu \alpha_{n} A_{2} y_{n}, \quad \forall n \geq 0,
\end{array}\right.
$$

where $\alpha_{n} \in(0,1]$ and $\lambda_{n} \in(0,2 \alpha]$ satisfy certain conditions. Let $A_{1}: H \rightarrow H$ be an inversestrongly monotone, $A_{2}: H \rightarrow H$ be a strongly monotone and Lipschitz continuous and $T: H \rightarrow H$ be a nonexpansive mapping, then the sequence converges strongly to the set solution of (1.2). 
In 2011, Yao et al. [19] studied new algorithms. For $x_{0} \in C$ chosen arbitrarily, let the sequence $\left\{x_{n}\right\}$ be generated iteratively by

$$
x_{n+1}=\beta_{n} x_{n}+\left(1-\beta_{n}\right) T P_{C}\left[I-\alpha_{n}(A-\gamma f)\right] x_{n}, \quad \forall n \geq 0,
$$

where the sequences $\left\{\alpha_{n}\right\}$ and $\left\{\beta_{n}\right\}$ are two sequences in $[0,1]$. Then $\left\{x_{n}\right\}$ converges strongly to $x^{\prime \prime} \in F(T)$ which is the unique solution of the variational inequality:

$$
\text { Find } x^{*} \in F(T) \text { such that }\left\langle(A-\gamma f) x^{*}, x-x^{*}\right\rangle \geq 0, \quad \forall x \in F(T) \text {. }
$$

Let $A: C \rightarrow H$ be a strongly positive linear bounded operator, $f: C \rightarrow H$ be a $\rho$ contraction and $T: C \rightarrow C$ be a nonexpansive mapping satisfying some conditions. The solution set of (1.3) is denoted by $\Omega:=V I(F(T), A-\gamma f)$.

Very recently, Marino and $\mathrm{Xu}[20]$ generated a sequence $\left\{x_{n}\right\}$ through the recursive formula

$$
x_{n+1}=\lambda_{n} f x_{n}+\left(1-\lambda_{n}\right)\left(\alpha_{n} V x_{n}+\left(1-\alpha_{n}\right) T x_{n}\right), \quad \forall n \geq 0,
$$

where $f$ is a contraction on $C$, the initial guess $x_{0} \in C$ is arbitrary and $\left\{\lambda_{n}\right\},\left\{\alpha_{n}\right\}$ are two sequences in $(0,1)$ and $T, V: C \rightarrow C$ are two nonexpansive self mappings. Strong convergence of the algorithm is proved under different circumstances of parameter selection.

In this paper, we introduce iterative algorithms and prove a strong convergence theorem for the following variational inequality over the triple hierarchical problem (1.4) below. Let $B: C \rightarrow C$ be a $\beta$-strongly monotone and $L$-Lipschitz continuous. Find $x^{*} \in \Omega$ such that

$$
\left\langle B x^{*}, x-x^{*}\right| \geq 0, \quad \forall x \in \Omega
$$

where $\Omega:=V I(F(T), A-\gamma f) \neq \emptyset, T$ is a nonexpansive mapping, $A: C \rightarrow H$ is a strongly positive linear bounded operator and $f: C \rightarrow H$ is a $\rho$-contraction. This solution set of (1.4) is denoted by $\Upsilon:=V I(\Omega, B):=V I(V I(F(T), A-\gamma f), B)$. Then the sequence $\left\{x_{n}\right\}$ strongly converges to the unique solution of (3.2) in Section 3 and we shall denote the set of such solutions by $\Theta:=V I(\Upsilon, I-\phi)$.

\section{Preliminaries}

Let $H$ be a real Hilbert space and $C$ be a nonempty closed convex subset of $H$. Recall that the (nearest point) projection $P_{C}$ from $H$ onto $C$ assigns, to each $x \in H$, the unique point in $P_{C} x \in C$ satisfying the property

$$
\left\|x-P_{C} x\right\|=\min _{y \in C}\|x-y\| .
$$

The following characterizes the projection $P_{C}$. We recall some lemmas which will be needed in the rest of this paper.

Lemma 2.1 The function $u \in C$ is a solution of the variational inequality (1.1) if and only if $u \in C$ satisfies the relation $u=P_{C}(u-\lambda A u)$ for all $\lambda>0$. 
Lemma 2.2 For a given $z \in H, u \in C, u=P_{C} z \Leftrightarrow\langle u-z, v-u\rangle \geq 0, \forall v \in C$.

It is well known that $P_{C}$ is a firmly nonexpansive mapping of $H$ onto $C$ and satisfies

$$
\left\|P_{C} x-P_{C} y\right\|^{2} \leq\left\langle P_{C} x-P_{C} y, x-y\right\rangle, \quad \forall x, y \in H .
$$

Moreover, $P_{C} x$ is characterized by the following properties: $P_{C} x \in C$ and for all $x \in H, y \in C$,

$$
\left\langle x-P_{C} x, y-P_{C} x\right\rangle \leq 0 .
$$

Lemma 2.3 The following inequality holds in an inner product space $H$ :

$$
\|x+y\|^{2} \leq\|x\|^{2}+2\langle y, x+y\rangle, \quad \forall x, y \in H .
$$

Lemma 2.4 [21] Let $C$ be a closed convex subset of a real Hilbert space $H$ and let $T$ : $C \rightarrow C$ be a nonexpansive mapping. Then $I-T$ is demiclosed at zero, that is, $x_{n} \rightarrow x$ and $x_{n}-T x_{n} \rightarrow 0$ imply $x=T x$.

Lemma 2.5 [22] Assume A is a strongly positive linear bounded operator on a Hilbert space $H$ with the coefficient $\bar{\gamma}>0$ and $0<\rho \leq\|A\|^{-1}$, then $\|I-\rho A\| \leq 1-\rho \bar{\gamma}$.

Lemma 2.6 [23] Each Hilbert space H satisfies Opial's condition, that is, for any sequence $\left\{x_{n}\right\} \subset H$ with $x_{n} \rightarrow x$, the inequality

$$
\liminf _{n \rightarrow \infty}\left\|x_{n}-x\right\|<\liminf _{n \rightarrow \infty}\left\|x_{n}-y\right\|
$$

holds for each $y \in H$ with $y \neq x$.

Lemma 2.7 [24] Let $\left\{x_{n}\right\}$ and $\left\{y_{n}\right\}$ be bounded sequences in a Banach space $X$ and let $\left\{\beta_{n}\right\}$ be a sequence in $[0,1]$ with $0<\liminf _{n \rightarrow \infty} \beta_{n} \leq \limsup _{n \rightarrow \infty} \beta_{n}<1$. Suppose $x_{n+1}=$ $\left(1-\beta_{n}\right) y_{n}+\beta_{n} x_{n}$ for all integers $n \geq 0$ and $\lim \sup _{n \rightarrow \infty}\left(\left\|y_{n+1}-y_{n}\right\|-\left\|x_{n+1}-x_{n}\right\|\right) \leq 0$. Then $\lim _{n \rightarrow \infty}\left\|y_{n}-x_{n}\right\|=0$.

Lemma 2.8 [10] Let $B: H \rightarrow H$ be $\beta$-strongly monotone and L-Lipschitz continuous and let $\mu \in\left(0, \frac{2 \beta}{L^{2}}\right)$. For $\lambda \in[0,1]$, define $T_{\lambda}: H \rightarrow H$ by $T_{\lambda}(x):=x-\lambda \mu B(x)$ for all $x \in H$. Then, for all $x, y \in H$,

$$
\left\|T_{\lambda}(x)-T_{\lambda}(y)\right\| \leq(1-\lambda \tau)\|x-y\|
$$

holds, where $\tau:=1-\sqrt{1-\mu\left(2 \beta-\mu L^{2}\right)} \in(0,1]$.

Lemma 2.9 [25] Assume $\left\{a_{n}\right\}$ is a sequence of nonnegative real numbers such that

$$
a_{n+1} \leq\left(1-\gamma_{n}\right) a_{n}+\delta_{n}, \quad \forall n \geq 0,
$$

where $\left\{\gamma_{n}\right\} \subset(0,1)$ and $\left\{\delta_{n}\right\}$ is a sequence in $\mathcal{R}$ such that

(i) $\sum_{n=1}^{\infty} \gamma_{n}=\infty$, 
(ii) $\lim \sup _{n \rightarrow \infty} \frac{\delta_{n}}{\gamma_{n}} \leq 0$ or $\sum_{n=1}^{\infty}\left|\delta_{n}\right|<\infty$.

Then $\lim _{n \rightarrow \infty} a_{n}=0$.

\section{Strong convergence theorem}

In this section, we introduce an iterative algorithm for solving the monotone variational inequality over a triple hierarchical problem.

Theorem 3.1 Let $H$ be a real Hilbert space, $C$ be a closed convex subset of H. Let $A: C \rightarrow H$ be a strongly positive linear bounded operator, $f: C \rightarrow H$ be a $\rho$-contraction, $\gamma$ be a positive real number such that $\frac{\bar{\gamma}-1}{\rho}<\gamma<\frac{\bar{\gamma}}{\rho}$. Let $T: C \rightarrow C$ be a nonexpansive mapping, $B: C \rightarrow C$ be a $\beta$-strongly monotone and L-Lipschitz continuous. Let $\phi: C \rightarrow C$ be a $k$-contraction mapping with $k \in[0,1)$. Assume that $\Upsilon:=V I(V I(F(T), A-\gamma f), B)$ is nonempty set. Suppose $\left\{x_{n}\right\}$ is a sequence generated by the following algorithm $x_{0} \in C$ arbitrarily:

$$
\left\{\begin{array}{l}
z_{n}=T P_{C}\left[I-\delta_{n}(A-\gamma f)\right] x_{n}, \\
y_{n}=\left(I-\mu \beta_{n} B\right) z_{n}, \\
x_{n+1}=\alpha_{n} \phi\left(x_{n}\right)+\left(1-\alpha_{n}\right) y_{n}, \quad \forall n \geq 0
\end{array}\right.
$$

where $\left\{\alpha_{n}\right\},\left\{\delta_{n}\right\} \subset[0,1]$. If $\mu \in\left(0, \frac{2 \beta}{L^{2}}\right)$ is used and if $\left\{\beta_{n}\right\} \subset(0,1]$ satisfies the following conditions:

(C1): $\sum_{n=1}^{\infty}\left|\delta_{n+1}-\delta_{n}\right|<\infty, \sum_{n=1}^{\infty} \delta_{n}=\infty$;

(C2): $\sum_{n=1}^{\infty}\left|\beta_{n+1}-\beta_{n}\right|<\infty$;

(C3): $\sum_{n=1}^{\infty}\left|\alpha_{n+1}-\alpha_{n}\right|<\infty, \lim _{n \rightarrow \infty} \alpha_{n}=0$;

(C4): $\delta_{n} \leq \beta_{n}$ and $\beta_{n} \leq \alpha_{n}$.

Then $\left\{x_{n}\right\}$ converges strongly to $x^{*} \in \Upsilon$, which is the unique solution of the variational inequality:

$$
\text { Find } x^{*} \in \Upsilon \text { such that }\left\langle(I-\phi) x^{*}, x-x^{*}\right\rangle \geq 0, \quad \forall x \in \Upsilon \text {. }
$$

Proof We will divide the proof into four steps.

Step 1 . We will show $\left\{x_{n}\right\}$ is bounded. For any $q \in \Upsilon$, we have

$$
\begin{aligned}
\left\|z_{n}-q\right\| & =\left\|T P_{C}\left[I-\delta_{n}(A-\gamma f)\right] x_{n}-T P_{C} q\right\| \\
& \leq\left\|\left[I-\delta_{n}(A-\gamma f)\right] x_{n}-q\right\| \\
& \leq \delta_{n}\left\|\gamma f\left(x_{n}\right)-\gamma f(q)\right\|+\delta_{n}\|\gamma f(q)-A q\|+\left\|I-\delta_{n} A\right\|\left\|x_{n}-q\right\| \\
& \leq \delta_{n} \gamma \rho\left\|x_{n}-q\right\|+\delta_{n}\|\gamma f(q)-A q\|+\left(1-\delta_{n} \bar{\gamma}\right)\left\|x_{n}-q\right\| \\
& =\left[1-(\bar{\gamma}-\gamma \rho) \delta_{n}\right]\left\|x_{n}-q\right\|+\delta_{n}\|\gamma f(q)-A q\| .
\end{aligned}
$$

By Lemma 2.8, it is found that

$$
\begin{aligned}
\left\|y_{n}-q\right\| & =\left\|\left(I-\mu \beta_{n} B\right) z_{n}-\left(I-\mu \beta_{n} B\right) q\right\| \\
& \leq\left(1-\beta_{n} \tau\right)\left\|z_{n}-q\right\| \\
& \leq\left(1-\beta_{n} \tau\right)\left\{\left[1-(\bar{\gamma}-\gamma \rho) \delta_{n}\right]\left\|x_{n}-q\right\|+\delta_{n}\|\gamma f(q)-A q\|\right\} .
\end{aligned}
$$


From (3.1), we get

$$
\begin{aligned}
& \left\|x_{n+1}-q\right\| \leq \alpha_{n}\left\|\phi\left(x_{n}\right)-\phi(q)\right\|+\alpha_{n}\|\phi(q)-q\|+\left(1-\alpha_{n}\right)\left\|y_{n}-q\right\| \\
& \leq \alpha_{n} k\left\|x_{n}-q\right\|+\alpha_{n}\|\phi(q)-\phi(q)\|+\left(1-\alpha_{n}\right)\left\|y_{n}-q\right\| \\
& \leq \alpha_{n} k\left\|x_{n}-q\right\|+\left(1-\alpha_{n}\right)\left(1-\beta_{n} \tau\right) \\
& \times\left\{\left[1-(\bar{\gamma}-\gamma \rho) \delta_{n}\right]\left\|x_{n}-q\right\|+\delta_{n}\|\gamma f(q)-A q\|\right\} \\
& \leq \alpha_{n}\left\|x_{n}-q\right\|+\left(1-\alpha_{n}\right)\left(1-\beta_{n} \tau\right)\left[1-(\bar{\gamma}-\gamma \rho) \delta_{n}\right]\left\|x_{n}-q\right\| \\
& +\left(1-\alpha_{n}\right)\left(1-\beta_{n} \tau\right) \delta_{n}\|\gamma f(q)-A q\| \\
& =\alpha_{n}\left\|x_{n}-q\right\|+\left(1-\alpha_{n}\right)\left[1-(\bar{\gamma}-\gamma \rho) \delta_{n}-\beta_{n} \tau+\beta_{n} \tau(\bar{\gamma}-\gamma \rho) \delta_{n}\right]\left\|x_{n}-q\right\| \\
& +\left(1-\alpha_{n}\right)\left(1-\beta_{n} \tau\right) \delta_{n}\|\gamma f(q)-A q\| \\
& =\alpha_{n}\left\|x_{n}-q\right\|+\left(1-\alpha_{n}\right)\left[1-\left\{(\bar{\gamma}-\gamma \rho) \delta_{n}+\beta_{n} \tau-\beta_{n} \tau(\bar{\gamma}-\gamma \rho) \delta_{n}\right\}\right]\left\|x_{n}-q\right\| \\
& +\left(1-\alpha_{n}\right)\left(1-\beta_{n} \tau\right) \delta_{n}\|\gamma f(q)-A q\| \\
& =\alpha_{n}\left\|x_{n}-q\right\| \\
& +\left[1-\alpha_{n}-\left\{(\bar{\gamma}-\gamma \rho) \delta_{n}+\beta_{n} \tau-\beta_{n} \tau(\bar{\gamma}-\gamma \rho) \delta_{n}\right\}\left(1-\alpha_{n}\right)\right]\left\|x_{n}-q\right\| \\
& +\left(1-\alpha_{n}\right)\left(1-\beta_{n} \tau\right) \delta_{n}\|\gamma f(q)-A q\| \\
& =\left[1-\left(1-\alpha_{n}\right)\left\{(\bar{\gamma}-\gamma \rho) \delta_{n}+\beta_{n} \tau-\beta_{n} \tau(\bar{\gamma}-\gamma \rho) \delta_{n}\right\}\right]\left\|x_{n}-q\right\| \\
& +\left(1-\alpha_{n}\right)\left(1-\beta_{n} \tau\right) \delta_{n}\|\gamma f(q)-A q\| \\
& =\left[1-\left(1-\alpha_{n}\right)\left\{(\bar{\gamma}-\gamma \rho) \delta_{n}\left(1-\beta_{n} \tau\right)+\beta_{n} \tau\right\}\right]\left\|x_{n}-q\right\| \\
& +\left(1-\alpha_{n}\right)\left(1-\beta_{n} \tau\right) \delta_{n}\|\gamma f(q)-A q\| \\
& =\left[1-\left(1-\alpha_{n}\right)(\bar{\gamma}-\gamma \rho) \delta_{n}\left(1-\beta_{n} \tau\right)-\left(1-\alpha_{n}\right) \beta_{n} \tau\right]\left\|x_{n}-q\right\| \\
& +\left(1-\alpha_{n}\right)\left(1-\beta_{n} \tau\right) \delta_{n}\|\gamma f(q)-A q\| \\
& =\left[1-\left(1-\alpha_{n}\right)(\bar{\gamma}-\gamma \rho) \delta_{n}\left(1-\beta_{n} \tau\right)\right]\left\|x_{n}-q\right\|-\left(1-\alpha_{n}\right) \beta_{n} \tau\left\|x_{n}-q\right\| \\
& +\left(1-\alpha_{n}\right)\left(1-\beta_{n} \tau\right) \delta_{n}\|\gamma f(q)-A q\| \\
& \leq\left[1-(\bar{\gamma}-\gamma \rho)\left(1-\alpha_{n}\right)\left(1-\beta_{n} \tau\right) \delta_{n}\right]\left\|x_{n}-q\right\| \\
& +(\bar{\gamma}-\gamma \rho)\left(1-\alpha_{n}\right)\left(1-\beta_{n} \tau\right) \delta_{n} \frac{\|\gamma f(q)-A q\|}{\bar{\gamma}-\gamma \rho} \\
& =\left(1-\sigma_{n}\right)\left\|x_{n}-q\right\|+\sigma_{n} \frac{\|\gamma f(q)-A q\|}{\bar{\gamma}-\gamma \rho},
\end{aligned}
$$

where $\sigma_{n}:=(\bar{\gamma}-\gamma \rho)\left(1-\alpha_{n}\right)\left(1-\beta_{n} \tau\right) \delta_{n}$. Then the mathematical induction implies that

$$
\left\|x_{n}-q\right\| \leq \max \left\{\left\|x_{0}-q\right\|, \frac{\|\gamma f(q)-A q\|}{\bar{\gamma}-\gamma \rho}\right\}, \quad \forall n \geq 0 .
$$

Therefore, $\left\{x_{n}\right\}$ is bounded and so are $\left\{y_{n}\right\},\left\{z_{n}\right\},\left\{A x_{n}\right\},\left\{B x_{n}\right\},\left\{\phi\left(x_{n}\right)\right\}$ and $\left\{f\left(x_{n}\right)\right\}$. 
Step 2. We claim that $\lim _{n \rightarrow \infty}\left\|x_{n+1}-x_{n}\right\|=0$ and $\lim _{n \rightarrow \infty}\left\|x_{n}-T x_{n}\right\|=0$. From (3.1), we have

$$
\begin{aligned}
\left\|z_{n+1}-z_{n}\right\|= & \left\|T P_{C}\left[I-\delta_{n+1}(A-\gamma f)\right] x_{n+1}-T P_{C}\left[I-\delta_{n}(A-\gamma f)\right] x_{n}\right\| \\
\leq & \left\|P_{C}\left[I-\delta_{n+1}(A-\gamma f)\right] x_{n+1}-P_{C}\left[I-\delta_{n}(A-\gamma f)\right] x_{n}\right\| \\
\leq & \left\|\left[I-\delta_{n+1}(A-\gamma f)\right] x_{n+1}-\left[I-\delta_{n}(A-\gamma f)\right] x_{n}\right\| \\
= & \| \delta_{n+1}\left(\gamma f\left(x_{n+1}\right)-\gamma f\left(x_{n}\right)\right)+\left(\delta_{n+1}-\delta_{n}\right) \gamma f\left(x_{n}\right)+\left(I-\delta_{n+1} A\right)\left(x_{n+1}-x_{n}\right) \\
& +\left(\delta_{n}-\delta_{n+1}\right) A x_{n} \| \\
\leq & \delta_{n+1} \gamma\left\|f\left(x_{n+1}\right)-f\left(x_{n}\right)\right\|+\left(1-\delta_{n+1} \bar{\gamma}\right)\left\|x_{n+1}-x_{n}\right\| \\
& +\left|\delta_{n+1}-\delta_{n}\right|\left(\left\|\gamma f\left(x_{n}\right)\right\|+\left\|A x_{n}\right\|\right) \\
\leq & \delta_{n+1} \gamma \rho\left\|x_{n+1}-x_{n}\right\|+\left(1-\delta_{n+1} \bar{\gamma}\right)\left\|x_{n+1}-x_{n}\right\| \\
& +\left|\delta_{n+1}-\delta_{n}\right|\left(\left\|\gamma f\left(x_{n}\right)\right\|+\left\|A x_{n}\right\|\right) \\
= & {\left[1-(\bar{\gamma}-\gamma \rho) \delta_{n+1}\right]\left\|x_{n+1}-x_{n}\right\|+\left|\delta_{n+1}-\delta_{n}\right|\left(\left\|\gamma f\left(x_{n}\right)\right\|+\left\|A x_{n}\right\|\right) }
\end{aligned}
$$

and

$$
\begin{aligned}
\left\|y_{n+1}-y_{n}\right\|= & \left\|\left(I-\mu \beta_{n+1} B\right) z_{n+1}-\left(I-\mu \beta_{n} B\right) z_{n}\right\| \\
\leq & \left\|\left(I-\mu \beta_{n+1} B\right) z_{n+1}-\left(I-\mu \beta_{n+1} B\right) z_{n}\right\| \\
& +\left\|\left(I-\mu \beta_{n+1} B\right) z_{n}-\left(I-\mu \beta_{n} B\right) z_{n}\right\| \\
\leq & \left(1-\beta_{n} \tau\right)\left\|z_{n+1}-z_{n}\right\|+\mu\left|\beta_{n+1}-\beta_{n}\right|\left\|B z_{n}\right\| .
\end{aligned}
$$

Using (3.5) and (3.6), we get

$$
\begin{aligned}
\left\|x_{n+2}-x_{n+1}\right\|= & \left\|\alpha_{n+1} \phi\left(x_{n+1}\right)+\left(1-\alpha_{n+1}\right) y_{n+1}-\alpha_{n} \phi\left(x_{n}\right)-\left(1-\alpha_{n}\right) y_{n}\right\| \\
\leq & \alpha_{n+1}\left\|\phi\left(x_{n+1}\right)-\phi\left(x_{n}\right)\right\|+\left|\alpha_{n+1}-\alpha_{n}\right|\left\|\phi\left(x_{n+1}\right)\right\|+\left(1-\alpha_{n+1}\right)\left\|y_{n+1}-y_{n}\right\| \\
& +\left|\alpha_{n+1}-\alpha_{n}\right|\left\|y_{n}\right\| \\
\leq & \alpha_{n+1} k\left\|x_{n+1}-x_{n}\right\|+\left|\alpha_{n+1}-\alpha_{n}\right|\left(\left\|\phi\left(x_{n+1}\right)\right\|+\left\|y_{n}\right\|\right) \\
& +\left(1-\alpha_{n+1}\right)\left\|y_{n+1}-y_{n}\right\| \\
\leq & \alpha_{n+1} k\left\|x_{n+1}-x_{n}\right\|+\left|\alpha_{n+1}-\alpha_{n}\right|\left(\left\|\phi\left(x_{n+1}\right)\right\|+\left\|y_{n}\right\|\right) \\
& +\left(1-\alpha_{n+1}\right)\left\{\left(1-\beta_{n} \tau\right)\left\|z_{n+1}-z_{n}\right\|+\mu\left|\beta_{n+1}-\beta_{n}\right|\left\|B z_{n}\right\|\right\} \\
= & \alpha_{n+1} k\left\|x_{n+1}-x_{n}\right\|+\left|\alpha_{n+1}-\alpha_{n}\right|\left(\left\|\phi\left(x_{n+1}\right)\right\|+\left\|y_{n}\right\|\right) \\
& +\left(1-\alpha_{n+1}\right)\left(1-\beta_{n} \tau\right)\left\|z_{n+1}-z_{n}\right\|+\left(1-\alpha_{n+1}\right) \mu\left|\beta_{n+1}-\beta_{n}\right|\left\|B z_{n}\right\| \\
\leq & \alpha_{n+1}\left\|x_{n+1}-x_{n}\right\|+\left|\alpha_{n+1}-\alpha_{n}\right|\left(\left\|\phi\left(x_{n+1}\right)\right\|+\left\|y_{n}\right\|\right) \\
& +\left(1-\alpha_{n+1}\right) \mu\left|\beta_{n+1}-\beta_{n}\right|\left\|B z_{n}\right\| \\
& +\left(1-\alpha_{n+1}\right)\left\{\left[1-(\bar{\gamma}-\gamma \rho) \delta_{n+1}\right]\left\|x_{n+1}-x_{n}\right\|\right. \\
& \left.+\left|\delta_{n+1}-\delta_{n}\right|\left(\left\|\gamma f\left(x_{n}\right)\right\|+\left\|A x_{n}\right\|\right)\right\}
\end{aligned}
$$




$$
\begin{aligned}
\leq & \alpha_{n+1}\left\|x_{n+1}-x_{n}\right\|+\left|\alpha_{n+1}-\alpha_{n}\right|\left(\left\|\phi\left(x_{n+1}\right)\right\|+\left\|y_{n}\right\|\right) \\
& +\left(1-\alpha_{n+1}\right) \mu\left|\beta_{n+1}-\beta_{n}\right|\left\|B z_{n}\right\| \\
& +\left(1-\alpha_{n+1}\right)\left[1-(\bar{\gamma}-\gamma \rho) \delta_{n+1}\right]\left\|x_{n+1}-x_{n}\right\| \\
& +\left(1-\alpha_{n+1}\right)\left|\delta_{n+1}-\delta_{n}\right|\left(\left\|\gamma f\left(x_{n}\right)\right\|+\left\|A x_{n}\right\|\right) \\
\leq & {\left[1-(\bar{\gamma}-\gamma \rho) \delta_{n+1}\left(1-\alpha_{n+1}\right)\right]\left\|x_{n+1}-x_{n}\right\| } \\
& +\left|\alpha_{n+1}-\alpha_{n}\right|\left(\left\|\phi\left(x_{n+1}\right)\right\|+\left\|y_{n}\right\|\right) \\
& +\mu\left|\beta_{n+1}-\beta_{n}\right|\left\|B z_{n}\right\|+\left|\delta_{n+1}-\delta_{n}\right|\left(\left\|\gamma f\left(x_{n}\right)\right\|+\left\|A x_{n}\right\|\right) \\
\leq & {\left[1-(\bar{\gamma}-\gamma \rho) \delta_{n+1}\left(1-\alpha_{n+1}\right)\right]\left\|x_{n+1}-x_{n}\right\| } \\
& +\left\{\left|\alpha_{n+1}-\alpha_{n}\right|+\left|\beta_{n+1}-\beta_{n}\right|+\left|\delta_{n+1}-\delta_{n}\right|\right\} M,
\end{aligned}
$$

where $M$ is some constant such that

$$
\sup _{n \geq 0}\left\{\left\|\phi\left(x_{n}\right)\right\|+\left\|y_{n}\right\|, \mu\left\|B z_{n}\right\|,\left\|\gamma f\left(x_{n}\right)\right\|+\left\|A x_{n}\right\|\right\} \leq M .
$$

From (C1)-(C3) and the boundedness of $\left\{x_{n}\right\},\left\{y_{n}\right\},\left\{A x_{n}\right\},\left\{B z_{n}\right\},\left\{\phi\left(x_{n}\right)\right\}$ and $\left\{f\left(x_{n}\right)\right\}$, by Lemma 2.9, we have

$$
\lim _{n \rightarrow \infty}\left\|x_{n+1}-x_{n}\right\|=0 .
$$

On the other hand, we note that

$$
\begin{aligned}
\left\|z_{n}-T x_{n}\right\| & =\left\|T P_{C}\left[I-\delta_{n}(A-\gamma f)\right] x_{n}-T x_{n}\right\| \\
& =\left\|T P_{C}\left[I-\delta_{n}(A-\gamma f)\right] x_{n}-T P_{C} x_{n}\right\| \\
& \leq\left\|\left[I-\delta_{n}(A-\gamma f)\right] x_{n}-x_{n}\right\| \\
& \left.\leq \delta_{n} \|(A-\gamma f)\right] x_{n} \|
\end{aligned}
$$

by (C3)-(C4) and it follows that

$$
\lim _{n \rightarrow \infty}\left\|z_{n}-T x_{n}\right\|=0 .
$$

From (3.1), we compute

$$
\begin{aligned}
\left\|x_{n+1}-z_{n}\right\| & =\left\|\alpha_{n} \phi\left(x_{n}\right)+\left(1-\alpha_{n}\right) y_{n}-z_{n}\right\| \\
& =\left\|\alpha_{n} \phi\left(x_{n}\right)+\left(1-\alpha_{n}\right)\left(I-\mu \beta_{n} B\right) z_{n}-z_{n}\right\| \\
& \leq \alpha_{n}\left\|\phi\left(x_{n}\right)-z_{n}\right\|+\left(1-\alpha_{n}\right)\left\|\left(I-\mu \beta_{n} B\right) z_{n}-z_{n}\right\| \\
& \leq \alpha_{n} k\left\|x_{n}-z_{n}\right\|+\alpha_{n}\left\|\phi\left(z_{n}\right)-z_{n}\right\|+\left(1-\alpha_{n}\right) \mu \beta_{n}\left\|B z_{n}\right\| .
\end{aligned}
$$

By (C3) and (C4), it follows that

$$
\lim _{n \rightarrow \infty}\left\|x_{n+1}-z_{n}\right\|=0 .
$$


Since

$$
\left\|x_{n}-T x_{n}\right\| \leq\left\|x_{n}-x_{n+1}\right\|+\left\|x_{n+1}-z_{n}\right\|+\left\|z_{n}-T x_{n}\right\| .
$$

By (3.7), (3.8) and (3.9), we obtain

$$
\lim _{n \rightarrow \infty}\left\|x_{n}-T x_{n}\right\|=0
$$

From (3.1), we compute

$$
\begin{aligned}
\left\|x_{n+1}-y_{n}\right\| & =\left\|\alpha_{n} \phi\left(x_{n}\right)+\left(1-\alpha_{n}\right) y_{n}-y_{n}\right\| \\
& =\left\|\alpha_{n} \phi\left(x_{n}\right)+y_{n}-\alpha_{n} y_{n}-y_{n}\right\| \\
& \leq \alpha_{n}\left\|\phi\left(x_{n}\right)-y_{n}\right\| .
\end{aligned}
$$

By (C3), it follows that

$$
\lim _{n \rightarrow \infty}\left\|x_{n+1}-y_{n}\right\|=0
$$

Since

$$
\left\|x_{n}-y_{n}\right\| \leq\left\|x_{n}-x_{n+1}\right\|+\left\|x_{n+1}-y_{n}\right\| \text {. }
$$

From (3.7) and (3.12), we obtain

$$
\lim _{n \rightarrow \infty}\left\|x_{n}-y_{n}\right\|=0
$$

Step 3. First, $\lim \sup _{n \rightarrow \infty}\left\langle u_{n}-x^{*}, \gamma f\left(x^{*}\right)-A x^{*}\right\rangle \leq 0$ is proven. Choose a subsequence $\left\{x_{n_{i}}\right\}$ of $\left\{x_{n}\right\}$ such that

$$
\limsup _{n \rightarrow \infty}\left\langle x_{n}-x^{*}, \gamma f\left(x^{*}\right)-A x^{*}\right\rangle=\lim _{i \rightarrow \infty}\left\langle x_{n_{i}}-x^{*}, \gamma f\left(x^{*}\right)-A x^{*}\right\rangle .
$$

The boundedness of $\left\{x_{n_{i}}\right\}$ implies the existences of a subsequence $\left\{x_{n_{i_{j}}}\right\}$ of $\left\{x_{n_{i}}\right\}$ and a point $\hat{x} \in H$ such that $\left\{x_{n_{i_{j}}}\right\}$ converges weakly to $\hat{x}$. We may assume, without loss of generality, that $\lim _{i \rightarrow \infty}\left\langle x_{n_{i}}, w\right\rangle=\langle\hat{x}, w\rangle, w \in H$. Assume $\hat{x} \neq T(\hat{x}) . \lim _{n \rightarrow \infty}\left\|x_{n}-T x_{n}\right\|=0$ with $F(T) \neq \emptyset$ guarantees that

$$
\begin{aligned}
& \liminf _{i \rightarrow \infty}\left\|x_{n_{i}}-\hat{x}\right\| \\
& \quad<\liminf _{i \rightarrow \infty}\left\|x_{n_{i}}-T(\hat{x})\right\| \\
& \quad=\liminf _{i \rightarrow \infty}\left\|x_{n_{i}}-T\left(x_{n_{i}}\right)+T\left(x_{n_{i}}\right)-T(\hat{x})\right\| \\
& \quad=\liminf _{i \rightarrow \infty}\left\|T\left(x_{n_{i}}\right)-T(\hat{x})\right\| \\
& \quad \leq \liminf _{i \rightarrow \infty}\left\|x_{n_{i}}-\hat{x}\right\|,
\end{aligned}
$$


which is a contradiction. Therefore, $\hat{x} \in F(T)$. From $x^{*} \in V I(F(T), A-\gamma f)$, we find

$$
\begin{aligned}
\limsup _{n \rightarrow \infty}\left\langle x_{n}-x^{*}, \gamma f\left(x^{*}\right)-A x^{*}\right\rangle & =\lim _{i \rightarrow \infty}\left\langle x_{n_{i}}-x^{*}, \gamma f\left(x^{*}\right)-A x^{*}\right\rangle \\
& =\left\langle\hat{x}-x^{*}, \gamma f\left(x^{*}\right)-A x^{*}\right\rangle \\
& \leq 0 .
\end{aligned}
$$

Setting $u_{n}=\left[I-\delta_{n}(A-\gamma f)\right] x_{n}$, by (C3)-(C4), we notice that

$$
\left\|u_{n}-x_{n}\right\| \leq \delta_{n}\|(A-\gamma f)\| \rightarrow 0 .
$$

Hence, we get

$$
\limsup _{n \rightarrow \infty}\left\langle u_{n}-x^{*}, \gamma f\left(x^{*}\right)-A x^{*}\right\rangle \leq 0
$$

Second, $\lim \sup _{n \rightarrow \infty}\left\langle x^{*}-x_{n+1}, B x^{\prime \prime}\right\rangle \leq 0$ is proven. $\lim _{n \rightarrow \infty}\left\|x_{n+1}-x_{n}\right\|=0$ guarantees the existences of a subsequence $\left\{x_{n_{k}+1}\right\}$ of $\left\{x_{n_{k}}\right\}$ and a point $\bar{x} \in H$ such that $\lim _{\sup _{n \rightarrow \infty}}\left\langle x^{*}-\right.$ $\left.x_{n+1}, B x^{*}\right\rangle=\lim _{k \rightarrow \infty}\left\langle x^{*}-x_{n_{k}+1}, B x^{*}\right\rangle$ and $\lim _{k \rightarrow \infty}\left\langle x_{n_{k}}, w\right\rangle=\lim _{k \rightarrow \infty}\left\langle x_{n_{k}+1}, w\right\rangle=\langle\bar{x}, w\rangle, w \in H$. By the same discussion as in the proof of $\hat{x} \in F(T)$, we have $\bar{x} \in F(T)$. Let $y \in F(T)$ be fixed arbitrarily. Then it follows that $T: C \rightarrow C$ is a nonexpansive mapping with $F(T) \neq \emptyset$, $A: C \rightarrow H$ is a strongly positive linear bounded operator and $f: C \rightarrow H$ is a contraction for all $n \in \mathbb{N}$. From (3.1),

$$
\begin{aligned}
\left\|z_{n}-y\right\| & =\left\|T P_{C} u_{n}-T P_{C} y\right\| \\
& \leq\left\|u_{n}-y\right\| .
\end{aligned}
$$

By (C3)-(C4), we observe that

$$
\begin{aligned}
\left\|u_{n}-y\right\| & =\left\|\left[I-\delta_{n}(A-\gamma f)\right] x_{n}-y\right\| \\
& \leq\left\|x_{n}-y\right\|+\delta_{n}\left\|(A-\gamma f) x_{n}\right\| \\
& \leq\left\|x_{n}-y\right\| .
\end{aligned}
$$

Using (3.15) and (3.16),

$$
\begin{aligned}
\left\|u_{n}-y\right\|^{2}= & \left\|\left[I-\delta_{n}(A-\gamma f)\right] x_{n}-y\right\|^{2} \\
= & \left\|\delta_{n}\left(\gamma f\left(x_{n}\right)-A y\right)+\left(I-\delta_{n} A\right)\left(x_{n}-y\right)\right\|^{2} \\
\leq & \left(1-\delta_{n} \bar{\gamma}\right)^{2}\left\|x_{n}-y\right\|^{2}+2 \delta_{n}\left\langle\gamma f\left(x_{n}\right)-A y, u_{n}-y\right\rangle \\
\leq & \left(1-2 \delta_{n} \bar{\gamma}+\delta_{n}^{2} \bar{\gamma}^{2}\right)\left\|x_{n}-y\right\|^{2}+2 \delta_{n} \gamma \rho\left\|x_{n}-y\right\|\left\|u_{n}-y\right\| \\
& +2 \delta_{n}\left(\gamma f(y)-A y, u_{n}-y\right\rangle \\
\leq & \left(1-2 \delta_{n} \bar{\gamma}+\delta_{n}^{2} \bar{\gamma}^{2}\right)\left\|x_{n}-y\right\|^{2}+2 \delta_{n} \gamma \rho\left\|x_{n}-y\right\|^{2}+2 \delta_{n}\left\langle\gamma f(y)-A y, u_{n}-y\right\rangle \\
= & {\left[1-2 \delta_{n}(\bar{\gamma}-\gamma \rho)\right]\left\|x_{n}-y\right\|^{2}+\delta_{n}^{2} \bar{\gamma}^{2}\left\|x_{n}-y\right\|^{2}+2 \delta_{n}\left\langle\gamma f(y)-A y, u_{n}-y\right\rangle, }
\end{aligned}
$$


which implies that

$$
\begin{aligned}
0 \leq & \left(\left\|x_{n}-y\right\|^{2}-\left\|u_{n}-y\right\|^{2}\right)-2 \delta_{n}(\bar{\gamma}-\gamma \rho)\left\|x_{n}-y\right\|^{2}+\delta_{n}^{2} \bar{\gamma}^{2}\left\|x_{n}-y\right\|^{2} \\
& +2 \delta_{n}\left(\gamma f(y)-A y, u_{n}-y\right\rangle \\
= & \left(\left\|x_{n}-y\right\|+\left\|u_{n}-y\right\|\right)\left(\left\|x_{n}-y\right\|-\left\|u_{n}-y\right\|\right) \\
& -2 \delta_{n}(\bar{\gamma}-\gamma \rho)\left\|x_{n}-y\right\|^{2}+\delta_{n}^{2} \bar{\gamma}^{2}\left\|x_{n}-y\right\|^{2} \\
& +2 \delta_{n}\left(\gamma f(y)-A y, u_{n}-y\right\rangle \\
\leq & M_{2}\left\|x_{n}-u_{n}\right\|-2 \delta_{n}(\bar{\gamma}-\gamma \rho)\left\|x_{n}-y\right\|^{2}+\delta_{n}^{2} \bar{\gamma}^{2}\left\|x_{n}-y\right\|^{2}+2 \delta_{n}\left\langle\gamma f(y)-A y, u_{n}-y\right\rangle,
\end{aligned}
$$

where $M_{2}:=\sup \left\{\left\|x_{n}-y\right\|+\left\|u_{n}-y\right\|: n \in \mathbb{N}\right\}<\infty$ for every $n \in \mathbb{N}$. By the weak convergence of $\left\{u_{n_{i}}\right\}$ to $\bar{x} \in F(T), \lim _{n \rightarrow \infty}\left\|u_{n}-x_{n}\right\|=0$ and (C3)-(C4), we get $\langle(\gamma f-A) y, \bar{x}-y\rangle \leq 0$ for all $y \in F(T)$. A mapping $A$ being a strongly positive linear bounded operator and $f$ being a contraction ensure $\langle(\gamma f-A) y, \bar{x}-y\rangle \leq 0$ for all $y \in F(T)$, that is, $\bar{x} \in V I(F(T), A-\gamma f)$. Thus, $x^{*} \in \operatorname{VI}(\operatorname{VI}(F(T), A-\gamma f), B)$, we have

$$
\begin{aligned}
\limsup _{n \rightarrow \infty}\left\langle x^{*}-x_{n}, B x^{*}\right\rangle & =\limsup _{i \rightarrow \infty}\left\langle x^{*}-x_{n_{i}}, B x^{*}\right\rangle \\
& =\left\langle x^{*}-\bar{x}, B x^{*}\right\rangle \\
& \leq 0 .
\end{aligned}
$$

From (3.13), we notice that

$$
\limsup _{n \rightarrow \infty}\left|x^{*}-y_{n}, B x^{*}\right\rangle \leq 0
$$

Third, $\lim \sup _{n \rightarrow \infty}\left\langle x_{n}-x^{*}, \phi\left(x^{*}\right)-x^{*}\right\rangle \leq 0$ is proven. Choose a subsequence $\left\{x_{n_{g}}\right\}$ of $\left\{x_{n}\right\}$ such that

$$
\limsup _{n \rightarrow \infty}\left\langle x_{n}-x^{*}, \phi\left(x^{*}\right)-x^{*}\right\rangle=\lim _{g \rightarrow \infty}\left\langle x_{n_{g}}-x^{*}, \phi\left(x^{*}\right)-x^{*}\right\rangle .
$$

The boundedness of $\left\{x_{n_{g}}\right\}$ implies the existence of a subsequence $\left\{x_{n_{g_{h}}}\right\}$ of $\left\{x_{n_{g}}\right\}$ and a point $\tilde{x} \in H$ such that $\left\{x_{n_{h}}\right\}$ converges weakly to $\tilde{x}$. By $\lim _{n \rightarrow \infty}\left\|x_{n+1}-x_{n}\right\|=0$, we have $\lim _{h \rightarrow \infty}\left\langle x_{n_{g_{h}+1}}, w\right\rangle=\langle\tilde{x}, w\rangle, w \in H$. We may assume, without loss of generality, that $\lim _{i \rightarrow \infty}\left\langle x_{n_{g}}, w\right\rangle=\langle\tilde{x}, w\rangle, w \in H$. Assume $\tilde{x} \neq T(\tilde{x}) . \lim _{n \rightarrow \infty}\left\|x_{n}-T x_{n}\right\|=0$ with $F(T) \neq \emptyset$ guarantees that

$$
\begin{aligned}
\liminf _{g \rightarrow \infty}\left\|x_{n_{g}}-\tilde{x}\right\| & <\liminf _{g \rightarrow \infty}\left\|x_{n_{g}}-T(\tilde{x})\right\| \\
& =\liminf _{g \rightarrow \infty}\left\|x_{n_{g}}-T\left(x_{n_{g}}\right)+T\left(x_{n_{g}}\right)-T(\tilde{x})\right\| \\
& =\liminf _{g \rightarrow \infty}\left\|T\left(x_{n_{g}}\right)-T(\tilde{x})\right\| \\
& \leq \liminf _{g \rightarrow \infty}\left\|x_{n_{g}}-\tilde{x}\right\| .
\end{aligned}
$$


This is a contradiction, that is, $\tilde{x} \in F(T)$. From $x^{*} \in V I(V I(V I(F(T), A-\gamma f), B), I-\phi)$, we find

$$
\begin{aligned}
& \limsup _{n \rightarrow \infty}\left\langle x_{n}-x^{*}, \phi\left(x^{*}\right)-x^{*}\right\rangle \\
& \quad=\lim _{g \rightarrow \infty}\left\langle x_{n_{g}}-x^{*}, \phi\left(x^{*}\right)-x^{*}\right\rangle \\
& \quad=\left\langle\tilde{x}-x^{*}, \phi\left(x^{*}\right)-x^{*}\right\rangle \\
& \quad \leq 0 .
\end{aligned}
$$

Step 4. Finally, we prove $\lim _{n \rightarrow \infty}\left\|x_{n}-x^{*}\right\|=0$. By Lemma 2.8, we compute

$$
\begin{aligned}
& \left\|x_{n+1}-x^{*}\right\|^{2}=\left\|\alpha_{n} \phi\left(x_{n}\right)+\left(1-\alpha_{n}\right) y_{n}-x^{*}\right\|^{2} \\
& =\left\|\alpha_{n}\left(\phi\left(x_{n}\right)-\phi\left(x^{*}\right)\right)+\alpha_{n}\left(\phi\left(x^{*}\right)-x^{*}\right)+\left(1-\alpha_{n}\right)\left(y_{n}-x^{*}\right)\right\|^{2} \\
& \leq \alpha_{n}\left\|\phi\left(x_{n}\right)-\phi\left(x^{*}\right)\right\|^{2}+\left(1-\alpha_{n}\right)\left\|y_{n}-x^{*}\right\|^{2}+2 \alpha_{n}\left\langle\phi\left(x^{\prime \prime}\right)-x^{\prime \prime}, x_{n+1}-x^{\prime \prime}\right\rangle \\
& \leq \alpha_{n} k^{2}\left\|x_{n}-x^{*}\right\|^{2}+\left(1-\alpha_{n}\right)\left\|\left(I-\mu \beta_{n} B\right) z_{n}-x^{*}\right\|^{2} \\
& +2 \alpha_{n}\left|\phi\left(x^{*}\right)-x^{*}, x_{n+1}-x^{*}\right\rangle \\
& =\alpha_{n} k^{2}\left\|x_{n}-x^{*}\right\|^{2}+\left(1-\alpha_{n}\right)\left\|\left(z_{n}-\mu \beta_{n} B z_{n}\right)-\left(x^{*}-\mu \beta_{n} B x^{*}\right)-\mu \beta_{n} B x^{*}\right\|^{2} \\
& +2 \alpha_{n}\left\langle\phi\left(x^{*}\right)-x^{*}, x_{n+1}-x^{*}\right\rangle \\
& \leq \alpha_{n} k^{2}\left\|x_{n}-x^{*}\right\|^{2}+\left(1-\alpha_{n}\right) \\
& \times\left\{\left\|\left(z_{n}-\mu \beta_{n} B z_{n}\right)-\left(x^{*}-\mu \beta_{n} B x^{*}\right)\right\|^{2}+2 \mu \beta_{n}\left(x^{*}-y_{n}, B x^{*}\right)\right\} \\
& +2 \alpha_{n}\left|\phi\left(x^{*}\right)-x^{*}, x_{n+1}-x^{*}\right\rangle \\
& \leq \alpha_{n} k^{2}\left\|x_{n}-x^{*}\right\|^{2}+\left(1-\alpha_{n}\right)\left(1-\tau \beta_{n}\right)^{2}\left\|z_{n}-x^{*}\right\|^{2}+2 \mu \beta_{n}\left\langle x^{*}-y_{n}, B x^{*}\right\rangle \\
& +2 \alpha_{n}\left\langle\phi\left(x^{*}\right)-x^{*}, x_{n+1}-x^{*}\right\rangle \\
& \leq \alpha_{n} k^{2}\left\|x_{n}-x^{*}\right\|^{2}+\left(1-\alpha_{n}\right)\left(1-\tau \beta_{n}\right)\left\|u_{n}-x^{*}\right\|^{2}+2 \mu \beta_{n}\left\langle x^{*}-y_{n}, B x^{*}\right\rangle \\
& +2 \alpha_{n}\left\langle\phi\left(x^{*}\right)-x^{* \prime}, x_{n+1}-x^{*}\right\rangle \\
& =\alpha_{n} k^{2}\left\|x_{n}-x^{*}\right\|^{2}+\left(1-\alpha_{n}\right)\left(1-\tau \beta_{n}\right)\left\|\left[I-\delta_{n}(A-\gamma f)\right] x_{n}-x^{*}\right\|^{2} \\
& +2 \mu \beta_{n}\left\langle x^{*}-y_{n}, B x^{*}\right\rangle+2 \alpha_{n}\left\langle\phi\left(x^{*}\right)-x^{*}, x_{n+1}-x^{*}\right\rangle \\
& =\alpha_{n} k^{2}\left\|x_{n}-x^{*}\right\|^{2}+\left(1-\alpha_{n}\right)\left(1-\tau \beta_{n}\right) \\
& \times\left\|\left(I-\delta_{n} A\right)\left(x_{n}-x^{*}\right)+\delta_{n}\left(\gamma f\left(x_{n}\right)-A x^{*}\right)\right\|^{2} \\
& +2 \mu \beta_{n}\left\langle x^{*}-y_{n}, B x^{*}\right\rangle+2 \alpha_{n}\left\langle\phi\left(x^{*}\right)-x^{*}, x_{n+1}-x^{*}\right\rangle \\
& \leq \alpha_{n} k^{2}\left\|x_{n}-x^{*}\right\|^{2}+\left(1-\alpha_{n}\right)\left(1-\tau \beta_{n}\right) \\
& \times\left\{\left(1-\delta_{n} \bar{\gamma}\right)^{2}\left\|x_{n}-x^{*}\right\|^{2}+2 \delta_{n}\left(\gamma f\left(x_{n}\right)-A x^{*}, u_{n}-x^{*}\right)\right\} \\
& +2 \mu \beta_{n}\left\langle x^{*}-y_{n}, B x^{*}\right\rangle+2 \alpha_{n}\left\langle\phi\left(x^{*}\right)-x^{*}, x_{n+1}-x^{*}\right\rangle \\
& \leq \alpha_{n} k\left\|x_{n}-x^{*}\right\|^{2}+\left(1-\alpha_{n}\right)\left(1-\tau \beta_{n}\right)\left\{\left(1-2 \delta_{n} \bar{\gamma}+\delta_{n}^{2} \bar{\gamma}^{2}\right)\left\|x_{n}-x^{*}\right\|^{2}\right. \\
& \left.+2 \delta_{n}\left\langle\gamma f\left(x_{n}\right)-\gamma f\left(x^{*}\right), u_{n}-x^{*}\right\rangle+2 \delta_{n}\left\langle\gamma f\left(x^{*}\right)-A x^{*}, u_{n}-x^{*}\right\rangle\right\}
\end{aligned}
$$




$$
\begin{aligned}
& +2 \mu \beta_{n}\left\langle x^{*}-y_{n}, B x^{*}\right\rangle+2 \alpha_{n}\left\langle\phi\left(x^{*}\right)-x^{*}, x_{n+1}-x^{*}\right\rangle \\
& \leq \alpha_{n} k\left\|x_{n}-x^{*}\right\|^{2}+\left(1-\alpha_{n}\right)\left(1-\tau \beta_{n}\right)\left\{\left(1-2 \delta_{n} \bar{\gamma}\right)\left\|x_{n}-x^{*}\right\|^{2}\right. \\
& +\delta_{n}^{2} \bar{\gamma}^{2}\left\|x_{n}-x^{*}\right\|^{2}+2 \delta_{n} \gamma \rho\left\|x_{n}-x^{*}\right\|\left\|u_{n}-x^{*}\right\| \\
& \left.+2 \delta_{n}\left\langle\gamma f\left(x^{*}\right)-A x^{*}, u_{n}-x^{*}\right\rangle\right\} \\
& +2 \mu \beta_{n}\left\langle x^{*}-y_{n}, B x^{*}\right\rangle+2 \alpha_{n}\left\langle\phi\left(x^{*}\right)-x^{*}, x_{n+1}-x^{*}\right\rangle \\
& \leq \alpha_{n}\left\|x_{n}-x^{*}\right\|^{2}+\left(1-\alpha_{n}\right)\left(1-\tau \beta_{n}\right)\left[1-2 \delta_{n}(\bar{\gamma}-\gamma \rho)\right]\left\|x_{n}-x^{*}\right\|^{2} \\
& +\left(1-\alpha_{n}\right)\left(1-\tau \beta_{n}\right) \delta_{n}^{2} \bar{\gamma}^{2}\left\|x_{n}-x^{*}\right\|^{2}+2 \delta_{n}\left\langle\gamma f\left(x^{*}\right)-A x^{*}, u_{n}-x^{*}\right\rangle \\
& +2 \mu \beta_{n}\left\langle x^{*}-y_{n}, B x^{*}\right\rangle+2 \alpha_{n}\left\langle\phi\left(x^{*}\right)-x^{*}, x_{n+1}-x^{*}\right\rangle \\
& =\alpha_{n}\left\|x_{n}-x^{*}\right\|^{2} \\
& +\left(1-\alpha_{n}\right)\left[1-2 \delta_{n}(\bar{\gamma}-\gamma \rho)-\tau \beta_{n}+\tau \beta_{n} 2 \delta_{n}(\bar{\gamma}-\gamma \rho)\right]\left\|x_{n}-x^{*}\right\|^{2} \\
& +\left(1-\alpha_{n}\right)\left(1-\tau \beta_{n}\right) \delta_{n}^{2} \bar{\gamma}^{2}\left\|x_{n}-x^{*}\right\|^{2}+2 \delta_{n}\left\langle\gamma f\left(x^{*}\right)-A x^{*}, u_{n}-x^{*}\right\rangle \\
& +2 \mu \beta_{n}\left\langle x^{*}-y_{n}, B x^{*}\right\rangle+2 \alpha_{n}\left\langle\phi\left(x^{*}\right)-x^{*}, x_{n+1}-x^{*}\right\rangle \\
& =\alpha_{n}\left\|x_{n}-x^{*}\right\|^{2} \\
& +\left(1-\alpha_{n}\right)\left[1-\left\{2 \delta_{n}(\bar{\gamma}-\gamma \rho)+\tau \beta_{n}-\tau \beta_{n} 2 \delta_{n}(\bar{\gamma}-\gamma \rho)\right\}\right]\left\|x_{n}-x^{n}\right\|^{2} \\
& +\left(1-\alpha_{n}\right)\left(1-\tau \beta_{n}\right) \delta_{n}^{2} \bar{\gamma}^{2}\left\|x_{n}-x^{*}\right\|^{2}+2 \delta_{n}\left\langle\gamma f\left(x^{*}\right)-A x^{*}, u_{n}-x^{*}\right\rangle \\
& +2 \mu \beta_{n}\left\langle x^{*}-y_{n}, B x^{*}\right\rangle+2 \alpha_{n}\left\langle\phi\left(x^{*}\right)-x^{*}, x_{n+1}-x^{*}\right\rangle \\
& =\left[1-\left(1-\alpha_{n}\right)\left\{2 \delta_{n}(\bar{\gamma}-\gamma \rho)+\tau \beta_{n}-\tau \beta_{n} 2 \delta_{n}(\bar{\gamma}-\gamma \rho)\right\}\right]\left\|x_{n}-x^{*}\right\|^{2} \\
& +\left(1-\alpha_{n}\right)\left(1-\tau \beta_{n}\right) \delta_{n}^{2} \bar{\gamma}^{2}\left\|x_{n}-x^{*}\right\|^{2}+2 \delta_{n}\left\langle\gamma f\left(x^{*}\right)-A x^{*}, u_{n}-x^{*}\right\rangle \\
& +2 \mu \beta_{n}\left\langle x^{*}-y_{n}, B x^{\prime \prime}\right\rangle+2 \alpha_{n}\left\langle\phi\left(x^{*}\right)-x^{\prime \prime}, x_{n+1}-x^{\prime \prime}\right\rangle \\
& =\left[1-\left(1-\alpha_{n}\right)\left\{2 \delta_{n}(\bar{\gamma}-\gamma \rho)\left(1-\tau \beta_{n}\right)+\tau \beta_{n}\right\}\right]\left\|x_{n}-x^{*}\right\|^{2} \\
& +\left(1-\alpha_{n}\right)\left(1-\tau \beta_{n}\right) \delta_{n}^{2} \bar{\gamma}^{2}\left\|x_{n}-x^{*}\right\|^{2}+2 \delta_{n}\left\langle\gamma f\left(x^{*}\right)-A x^{*}, u_{n}-x^{*}\right\rangle \\
& +2 \mu \beta_{n}\left\langle x^{*}-y_{n}, B x^{*}\right\rangle+2 \alpha_{n}\left\langle\phi\left(x^{*}\right)-x^{*}, x_{n+1}-x^{*}\right\rangle \\
& =\left[1-\left(1-\alpha_{n}\right) 2 \delta_{n}(\bar{\gamma}-\gamma \rho)\left(1-\tau \beta_{n}\right)\right]\left\|x_{n}-x^{*}\right\|^{2}-\left(1-\alpha_{n}\right) \tau \beta_{n}\left\|x_{n}-x^{*}\right\|^{2} \\
& +\left(1-\alpha_{n}\right)\left(1-\tau \beta_{n}\right) \delta_{n}^{2} \bar{\gamma}^{2}\left\|x_{n}-x^{*}\right\|^{2}+2 \delta_{n}\left\langle\gamma f\left(x^{*}\right)-A x^{*}, u_{n}-x^{*}\right\rangle \\
& +2 \mu \beta_{n}\left\langle x^{*}-y_{n}, B x^{*}\right\rangle+2 \alpha_{n}\left\langle\phi\left(x^{*}\right)-x^{*}, x_{n+1}-x^{*}\right\rangle \\
& \leq\left[1-2(\bar{\gamma}-\gamma \rho)\left(1-\alpha_{n}\right)\left(1-\tau \beta_{n}\right) \delta_{n}\right]\left\|x_{n}-x^{*}\right\|^{2} \\
& +\left(1-\alpha_{n}\right)\left(1-\tau \beta_{n}\right) \delta_{n}^{2} \bar{\gamma}^{2}\left\|x_{n}-x^{*}\right\|^{2}+2 \delta_{n}\left\langle\gamma f\left(x^{*}\right)-A x^{*}, u_{n}-x^{*}\right\rangle \\
& +2 \mu \beta_{n}\left\langle x^{*}-y_{n}, B x^{*}\right\rangle+2 \alpha_{n}\left\langle\phi\left(x^{*}\right)-x^{*}, x_{n+1}-x^{*}\right\rangle \text {. }
\end{aligned}
$$

Since $\left\{x_{n}\right\},\left\{A x_{n}\right\},\left\{B x_{n}\right\},\left\{\phi\left(x_{n}\right)\right\}$ and $\left\{f\left(x_{n}\right)\right\}$ are all bounded, we can choose a constant $M_{1}>0$ such that

$$
\sup _{n} \frac{1}{\bar{\gamma}-\gamma \rho}\left\{\frac{\delta_{n} \bar{\gamma}^{2}}{2}\left\|x_{n}-x^{*}\right\|^{2}\right\} \leq M_{1}
$$


It follows that

$$
\begin{aligned}
\left\|x_{n+1}-x^{*}\right\|^{2} \leq & {\left[1-2(\bar{\gamma}-\gamma \rho)\left(1-\alpha_{n}\right)\left(1-\tau \beta_{n}\right) \delta_{n}\right]\left\|x_{n}-x^{*}\right\|^{2} } \\
& +2(\bar{\gamma}-\gamma \rho)\left(1-\alpha_{n}\right)\left(1-\tau \beta_{n}\right) \delta_{n} \varsigma_{n},
\end{aligned}
$$

where

$$
\begin{aligned}
\varsigma_{n}= & \delta_{n} M_{1}+\frac{1}{(\bar{\gamma}-\gamma \rho)\left(1-\alpha_{n}\right)\left(1-\tau \beta_{n}\right)}\left\langle\gamma f\left(x^{*}\right)-A x^{*}, u_{n}-x^{*}\right\rangle \\
& +\frac{\mu \beta_{n}}{(\bar{\gamma}-\gamma \rho)\left(1-\alpha_{n}\right)\left(1-\tau \beta_{n}\right) \delta_{n}}\left\langle x^{*}-y_{n}, B x^{*}\right\rangle \\
& +\frac{\alpha_{n}}{(\bar{\gamma}-\gamma \rho)\left(1-\alpha_{n}\right)\left(1-\tau \beta_{n}\right) \delta_{n}}\left\langle\phi\left(x^{*}\right)-x^{*}, x_{n+1}-x^{*}\right\rangle .
\end{aligned}
$$

By (3.14), (3.17), (3.18) and (C3)-(C4), we get $\lim \sup _{n \rightarrow \infty} \varsigma_{n} \leq 0$. Applying Lemma 2.9, we can conclude that $x_{n} \rightarrow x^{*}$. This completes the proof.

\section{An example}

Next, the following example shows that all the conditions of Theorem 3.1 are satisfied.

Example 4.1 For instance, let $\alpha_{n}=\frac{1}{n}, \beta_{n}=\frac{1}{2 n}$ and $\delta_{n}=\frac{1}{3 n}$. We will show that the condition $(\mathrm{C} 1)$ is achieved. Then, clearly, the sequence $\left\{\delta_{n}\right\}$

$$
\sum_{n=1}^{\infty} \delta_{n}=\sum_{n=1}^{\infty} \frac{1}{3 n}=\infty
$$

and

$$
\begin{aligned}
\sum_{n=1}^{\infty}\left|\delta_{n+1}-\delta_{n}\right| & =\sum_{n=1}^{\infty}\left|\frac{1}{3(n+1)}-\frac{1}{3 n}\right| \\
& \leq\left|\frac{1}{3 \cdot 1}-\frac{1}{3 \cdot 2}\right|+\left|\frac{1}{3 \cdot 2}-\frac{1}{3 \cdot 3}\right|+\left|\frac{1}{3 \cdot 3}-\frac{1}{3 \cdot 4}\right|+\cdots \\
& =\frac{1}{3} .
\end{aligned}
$$

The sequence $\left\{\delta_{n}\right\}$ satisfies the condition $(\mathrm{C} 1)$.

Next, we will show that the condition $(\mathrm{C} 2)$ is achieved. We compute

$$
\begin{aligned}
\sum_{n=1}^{\infty}\left|\beta_{n+1}-\beta_{n}\right| & =\sum_{n=1}^{\infty}\left|\frac{1}{2(n+1)}-\frac{1}{2 n}\right| \\
& \leq\left|\frac{1}{2 \cdot 1}-\frac{1}{2 \cdot 2}\right|+\left|\frac{1}{2 \cdot 2}-\frac{1}{2 \cdot 3}\right|+\left|\frac{1}{2 \cdot 3}-\frac{1}{2 \cdot 4}\right|+\cdots \\
& =\frac{1}{2} .
\end{aligned}
$$

The sequence $\left\{\beta_{n}\right\}$ satisfies the condition (C2). 
Next, we will show that the condition (C3) is achieved. We compute

$$
\begin{aligned}
\sum_{n=1}^{\infty}\left|\alpha_{n+1}-\alpha_{n}\right| & =\sum_{n=1}^{\infty}\left|\frac{1}{n+1}-\frac{1}{n}\right| \\
& \leq\left|\frac{1}{1}-\frac{1}{2}\right|+\left|\frac{1}{2}-\frac{1}{3}\right|+\left|\frac{1}{3}-\frac{1}{4}\right|+\cdots \\
& =1
\end{aligned}
$$

and

$$
\lim _{n \rightarrow \infty} \alpha_{n}=\lim _{n \rightarrow \infty} \frac{1}{n}=0
$$

The sequence $\left\{\alpha_{n}\right\}$ satisfies the condition (C3).

Finally, we will show that the condition (C4) is achieved.

$$
\frac{1}{3 n}<\frac{1}{2 n} \quad \text { and } \quad \frac{1}{2 n}<\frac{1}{n} .
$$

Corollary 4.2 Let $H$ be a real Hilbert space, $C$ be a closed convex subset of $H$. Let $A: C \rightarrow$ $H$ be inverse-strongly monotone. Let $T: C \rightarrow C$ be a nonexpansive mapping. Let $B: C \rightarrow C$ be $\beta$-strongly monotone and L-Lipschitz continuous. Assume that $\operatorname{VI}(F(T), A)$ is nonempty set. Suppose $\left\{x_{n}\right\}$ is a sequence generated by the following algorithm $x_{0} \in C$ arbitrarily:

$$
\left\{\begin{array}{l}
z_{n}=T\left(I-\delta_{n} A\right) x_{n}, \\
y_{n}=\left(I-\mu \beta_{n} B\right) z_{n}, \\
x_{n+1}=\left(1-\alpha_{n}\right) y_{n}, \quad \forall n \geq 0
\end{array}\right.
$$

$\left\{\alpha_{n}\right\},\left\{\delta_{n}\right\} \subset[0,1]$. If $\mu \in\left(0, \frac{2 \beta}{L^{2}}\right)$ is used and if $\left\{\beta_{n}\right\} \subset(0,1]$ satisfies the following conditions:

(C1): $\sum_{n=1}^{\infty}\left|\delta_{n+1}-\delta_{n}\right|<\infty, \sum_{n=1}^{\infty} \delta_{n}=\infty$;

(C2): $\sum_{n=1}^{\infty}\left|\beta_{n+1}-\beta_{n}\right|<\infty$;

(C3): $\sum_{n=1}^{\infty}\left|\alpha_{n+1}-\alpha_{n}\right|<\infty, \lim _{n \rightarrow \infty} \alpha_{n}=0$;

(C4): $\delta_{n} \leq \beta_{n}$ and $\beta_{n} \leq \alpha_{n}$.

Then $\left\{x_{n}\right\}$ converges strongly to $x^{*} \in V I(F(T), A)$, which is the unique solution of the variational inequality:

$$
\text { Find } x^{*} \in V I(F(T), A) \text { such that }\left\langle B x^{*}, x-x^{*}\right\rangle \geq 0, \quad \forall x \in V I(F(T), A) \text {. }
$$

Proof Putting $P_{C}$ is the identity and $f, \phi \equiv 0$ in Theorem 3.1, we can obtain the desired conclusion immediately.

Remark 4.3 Corollary 4.2 generalizes and improves the results of Iiduka [14].

Corollary 4.4 Let $H$ be a real Hilbert space, $C$ be a closed convex subset of $H$. Let $A: C \rightarrow H$ be a strongly positive linear bounded operator, $f: C \rightarrow H$ be a $\rho$-contraction, $\gamma$ be a positive real number such that $\frac{\bar{\gamma}-1}{\rho}<\gamma<\frac{\bar{\gamma}}{\rho}$. Let $T: C \rightarrow C$ be a nonexpansive mapping. Assume that $\Omega$ is nonempty set. Suppose $\left\{x_{n}\right\}$ is a sequence generated by the following 
algorithm $x_{0} \in C$ arbitrarily:

$$
\left\{\begin{array}{l}
z_{n}=T P_{C}\left[I-\delta_{n}(A-\gamma f)\right] x_{n}, \\
y_{n}=\left(I-\mu \beta_{n} B\right) z_{n}, \\
x_{n+1}=\alpha_{n}\left(x_{n}\right)+\left(1-\alpha_{n}\right) y_{n}, \quad \forall n \geq 0,
\end{array}\right.
$$

where $\left\{\alpha_{n}\right\},\left\{\delta_{n}\right\} \subset[0,1]$. If $\mu \in\left(0, \frac{2 \beta}{L^{2}}\right)$ is used and if $\left\{\beta_{n}\right\} \subset(0,1]$ satisfies the following conditions:

(C1): $\sum_{n=1}^{\infty}\left|\delta_{n+1}-\delta_{n}\right|<\infty, \sum_{n=1}^{\infty} \delta_{n}=\infty$;

(C2): $\sum_{n=1}^{\infty}\left|\beta_{n+1}-\beta_{n}\right|<\infty$;

(C3): $\sum_{n=1}^{\infty}\left|\alpha_{n+1}-\alpha_{n}\right|<\infty, \lim _{n \rightarrow \infty} \alpha_{n}=0$;

(C4): $\delta_{n} \leq \beta_{n}$ and $\beta_{n} \leq \alpha_{n}$.

Then $\left\{x_{n}\right\}$ converges strongly to $x^{\prime \prime} \in \Omega$, which is the unique solution of the variational inequality:

$$
\text { Find } x^{*} \in \Omega \text { such that }\left\langle B x^{*}, x-x^{*}\right\rangle \geq 0, \quad \forall x \in \Omega \text {. }
$$

Proof Putting $\phi$ is the identity in Theorem 3.1, we can obtain the desired conclusion immediately.

Remark 4.5 Corollary 4.4 generalizes and improves the results of Marino and Xu [20].

\section{Competing interests}

The authors declare that they have no competing interests.

\section{Authors' contributions}

Both authors read and approved the final manuscript.

\section{Author details}

'Department of Mathematics, Faculty of Science, King Mongkut's University of Technology Thonburi (KMUTT), Bangmod, Thung Khru, Bangkok, 10140, Thailand. ${ }^{2}$ Department of Mathematics, Faculty of Science and Agricultural Technology,

Rajamangala University of Technology Lanna, Phan, Chiang Rai, 57120, Thailand.

\section{Acknowledgements}

The authors thank to the Higher Education Research Promotion and National Research University Project of Thailand, the Office of the Higher Education Commission for financial support during the preparation of this paper.

Received: 10 May 2012 Accepted: 13 November 2012 Published: 3 December 2012

\section{References}

1. Hartman, P, Stampacchia, G: On some nonlinear elliptic differential functional equations. Acta Math. 115, 271-310 (1966)

2. Cianciaruso, F, Marino, G, Muglia, L, Yao, Y: On a two-step algorithm for hierarchical fixed point problems and variational inequalities. J. Inequal. Appl. 2009, Article ID 208692 (2009)

3. Yao, JC, Chadli, O: Pseudomonotone complementarity problems and variational inequalities. In: Crouzeix, JP, Haddjissas, N, Schaible, S (eds.) Handbook of Generalized Convexity and Monotonicity, pp. 501-558 (2005)

4. Kirk, WA: Fixed point theorem for mappings which do not increase distance. Am. Math. Mon. 72, 1004-1006 (1965)

5. Combettes, PL: A block-iterative surrogate constraint splitting method for quadratic signal recovery. IEEE Trans. Signal Process. 51(7), 1771-1782 (2003)

6. Hirstoaga, SA: Iterative selection method for common fixed point problems. J. Math. Anal. Appl. 324, 1020-1035 (2006)

7. liduka, $\mathrm{H}$, Yamada, I: A subgradient-type method for the equilibrium problem over the fixed point set and its applications. Optimization 58(2), 251-261 (2009)

8. Slavakis, K, Yamada, I: Robust wideband beamforming by the hybrid steepest descent method. IEEE Trans. Signal Process. 55(9), 4511-4522 (2007)

9. Slavakis, K, Yamada, I, Sakaniwa, K: Computation of symmetric positive definite Toeplitz matrices by the hybrid steepest descent method. Signal Process. 83, 1135-1140 (2003) 
10. Yamada, I: The hybrid steepest descent method for the variational inequality problems over the intersection of fixed point sets of nonexpansive mappings. In: Butnariu, D, Censor, Y, Reich, S (eds.) Inherently Paralle Algorithms for Feasibllity and Optimization and Their Applications, pp. 473-504. Elsevier, Amsterdam (2001)

11. Yao, Y, Cho, YJ, Liou, Y-C: Iterative algorithms for hierarchical fixed points problems and variational inequalities. Math. Comput. Model. 52, 1697-1705 (2010)

12. Yamada, I, Ogura, N, Shirakawa, N: A numerically robust hybrid steepest descent method for the convexly constrained generalized inverse problems. In: Nashed, Z, Scherzer, O (eds.) Inverse Problems, Image Analysis, and Medical Imaging. Contemp. Math., vol. 313, pp. 269-305. Amer. Math. Soc., Providence (2002)

13. Yamada, I, Ogura, N: Hybrid steepest descent method for variational inequality problem over the fixed point set of certain quasi-nonexpansive mapping. Numer. Funct. Anal. Optim. 25, 619-655 (2004)

14. liduka, $\mathrm{H}$ : Strong convergence for an iterative method for the triple-hierarchical constrained optimization problem. Nonlinear Anal. 71, e1292-e1297 (2009)

15. liduka, H: Iterative algorithm for solving triple-hierarchical constrained optimization problem. J. Optim. Theory Appl. 148(3), 580-592 (2011)

16. Jitpeera, T, Kumam, P: A new explicit triple hierarchical problem over the set of fixed point and generalized mixed equilibrium problem. J. Inequal. Appl. 2012, 82 (2012). doi:10.1186/1029-242X-2012-82

17. Jitpeera, T, Kumam, P: Algorithms for solving the variational inequality problem over the triple hierarchical problem. Abstr. Appl. Anal. 2012, Article ID 827156 (2012). doi:10.1155/2012/827156

18. Wairojjana, N, Kumam, P: General iterative algorithms for hierarchical fixed points approach to variational inequalities J. Appl. Math. 2012, Article ID 174318 (2012). doi:10.1155/2012/174318

19. Yao, Y, Liou, Y-C, Kang, SM: Algorithms construction for variational inequalities. Fixed Point Theory Appl. 2011, Article ID 794203 (2011). doi:10.1155/2011/794203

20. Marino, G, Xu, H-K: Explicit hierarchical fixed point approach to variational inequalities. J. Optim. Theory Appl. 149(1), 61-78 (2011)

21. Browder, FE: Nonlinear operators and nonlinear equations of evolution in Banach spaces. Proc. Symp. Pure Math. 18, 78-81 (1976)

22. Marino, G, Xu, HK: A general iterative method for nonexpansive mapping in Hilbert space. J. Math. Anal. Appl. 318, 43-52 (2006)

23. Opial, Z: Weak convergence of the sequence of successive approximations for nonexpansive mappings. Bull. Am. Math. Soc. 73, 595-597 (1967)

24. Suzuki, T: Strong convergence of Krasnoselskii and Mann's type sequences for one-parameter nonexpansive semigroups without Bochner integrals. J. Math. Anal. Appl. 305(1), 227-239 (2005)

25. Xu, HK: Iterative algorithms for nonlinear operators. J. Lond. Math. Soc. 66, 240-256 (2002)

doi:10.1186/1029-242X-2012-280

Cite this article as: Wairojjana et al.: The hybrid steepest descent method for solving variational inequality over triple hierarchical problems. Journal of Inequalities and Applications 2012 2012:280.

\section{Submit your manuscript to a SpringerOpen ${ }^{\circ}$ journal and benefit from:}

- Convenient online submission

- Rigorous peer review

- Immediate publication on acceptance

- Open access: articles freely available online

- High visibility within the field

- Retaining the copyright to your article 